\title{
280 one-opposition near-Earth asteroids recovered by the EURONEAR with the Isaac Newton Telescope ${ }^{\star}$
}

\author{
O. Vaduvescu ${ }^{1,2,3}$, L. Hudin ${ }^{4}$, T. Mocnik ${ }^{1}$, F. Char ${ }^{5}$, A. Sonka ${ }^{6}$, V. Tudor ${ }^{1}$, I. Ordonez-Etxeberria ${ }^{1,7}$, \\ M. Díaz Alfaro ${ }^{1,8}$, R. Ashley ${ }^{1}$, R. Errmann ${ }^{1}$, P. Short ${ }^{1}$, A. Moloceniuc ${ }^{9}$, R. Cornea ${ }^{9}$, V. Inceu ${ }^{10}$, D. Zavoianu ${ }^{11}$, \\ M. Popescu ${ }^{6,12}$, L. Curelaru9 ${ }^{9}$, S. Mihalea ${ }^{9}$, A.-M. Stoian ${ }^{13}$, A. Boldea ${ }^{14,15}$, R. Toma ${ }^{16,9}$, L. Fields ${ }^{16}$, V. Grigore ${ }^{9}$, \\ H. Stoev ${ }^{1}$, F. Lopez-Martinez ${ }^{1,17}$, N. Humphries ${ }^{1}$, P. Sowicka ${ }^{1}{ }^{18}$, Y. Ramanjooloo ${ }^{1}$, A. Manilla-Robles ${ }^{1}$, \\ F. C. Riddick ${ }^{1}$, F. Jimenez-Lujan ${ }^{1}$, J. Mendez ${ }^{1}$, F. Aceituno ${ }^{19}$, A. Sota ${ }^{19}$, D. Jones ${ }^{2,3}$, S. Hidalgo ${ }^{2,3}$, S. Murabito ${ }^{2,3}$, \\ I. Oteo ${ }^{20,21}$, A. Bongiovanni ${ }^{2,3}$, O. Zamora ${ }^{2,3}$, S. Pyrzas ${ }^{2,3,22}$, R. Génova-Santos ${ }^{2,3}$, J. Font ${ }^{2,3}$, A. Bereciartua ${ }^{2,3}$, \\ I. Perez-Fournon ${ }^{2,3}$, C. E. Martínez-Vázquez ${ }^{2,3}$, M. Monellii ${ }^{2,3}$, L. Cicuendez ${ }^{2,3}$, L. Monteagudo ${ }^{2,3}$, I. Agulli², 3 , \\ H. Bouy ${ }^{23,24}$, N. Huélamo ${ }^{24}$, M. Monguió ${ }^{25}$, B. T. Gänsicke ${ }^{26}$, D. Steeghs ${ }^{26}$, N. P. Gentile-Fusillo ${ }^{26}$, M. A. Hollands ${ }^{26}$, \\ O. Toloza $^{26}$, C. J. Manser ${ }^{26}$, V. Dhillon ${ }^{27,2}$, D. Sahman ${ }^{27}$, A. Fitzsimmons ${ }^{28}$, A. McNeill ${ }^{28}$, A. Thompson ${ }^{28}$

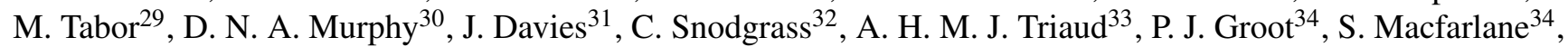 \\ R. Peletier ${ }^{35}$, S. Sen ${ }^{35}$, T. İkiz ${ }^{35}$, H. Hoekstra ${ }^{36}$, R. Herbonnet $^{36}$, F. Köhlinger ${ }^{36}$, R. Greimel ${ }^{37}$, A. Afonso ${ }^{38}$, \\ Q. A. Parker ${ }^{39,40}$, A. K. H. Kong ${ }^{41}$, C. Bassa ${ }^{42}$, and Z. Pleunis ${ }^{43}$
}

(Affiliations can be found after the references)

Received 28 August 2017 / Accepted 11 October 2017

ABSTRACT

Context. One-opposition near-Earth asteroids (NEAs) are growing in number, and they must be recovered to prevent loss and mismatch risk, and to improve their orbits, as they are likely to be too faint for detection in shallow surveys at future apparitions.

Aims. We aimed to recover more than half of the one-opposition NEAs recommended for observations by the Minor Planet Center (MPC) using the Isaac Newton Telescope (INT) in soft-override mode and some fractions of available D-nights. During about $130 \mathrm{~h}$ in total between 2013 and 2016, we targeted 368 NEAs, among which 56 potentially hazardous asteroids (PHAs), observing 437 INT Wide Field Camera (WFC) fields and recovering 280 NEAs (76\% of all targets).

Methods. Engaging a core team of about ten students and amateurs, we used the THELI, Astrometrica, and the Find_Orb software to identify all moving objects using the blink and track-and-stack method for the faintest targets and plotting the positional uncertainty ellipse from NEODyS. Results. Most targets and recovered objects had apparent magnitudes centered around $V \sim 22.8$ mag, with some becoming as faint as $V \sim 24$ mag. One hundred and three objects (representing 28\% of all targets) were recovered by EURONEAR alone by Aug. 2017. Orbital arcs were prolonged typically from a few weeks to a few years; our oldest recoveries reach 16 years. The $\mathrm{O}-\mathrm{C}$ residuals for our 1854 NEA astrometric positions show that most measurements cluster closely around the origin. In addition to the recovered NEAs, 22000 positions of about 3500 known minor planets and another 10000 observations of about 1500 unknown objects (mostly main-belt objects) were promptly reported to the MPC by our team. Four new NEAs were discovered serendipitously in the analyzed fields and were promptly secured with the INT and other telescopes, while two more NEAs were lost due to extremely fast motion and lack of rapid follow-up time. They increase the counting to nine NEAs discovered by the EURONEAR in 2014 and 2015.

Conclusions. Targeted projects to recover one-opposition NEAs are efficient in override access, especially using at least two-meter class and preferably larger field telescopes located in good sites, which appear even more efficient than the existing surveys.

Key words. astrometry - minor planets, asteroids: general

\section{Introduction}

The recovery of an asteroid is defined as an observation made during a second apparition (best-visibility period, which typically takes place around a new opposition) following the discovery (Boattini 2000). The recovery of poorly observed asteroids and especially near-Earth asteroids (NEAs) and near-Earth objects (NEOs) is a very important task to prevent object loss and mispairing, and to improve the orbits and dynamical evolution.

Very few papers have so far described targeted recovery and follow-up programs of NEAs. We mention here the pioneering efforts of Tatum (1994), who used three telescopes in Canada (including the DAO $1.85 \mathrm{~m}$ ) to follow up 38 NEAs and recover

\footnotetext{
* Table 2 is only available at the CDS via anonymous ftp to cdsarc.u-strasbg. fr (130.79.128.5) or via http://cdsarc.u-strasbg.fr/viz-bin/qcat?J/A+A/609/A105
}

2 NEAs during 1992. Boattini (2000) presented some statistics based on a sample of multi-opposition NEAs, sorting recoveries into four classes that included new observations and data mining of existing image archives and concluding that planning telescope observations is the best way to recover NEAs. Tichá (2000, 2002) presented recoveries of 21 NEAs over four and half years (1997-2001) using the $0.57 \mathrm{~m}$ telescope at Klet' observatory in Slovakia. Since 2002, the follow-up (mainly) and recovery efforts at Klet' have been improved through the KLENOT program, using a dedicated $1.06 \mathrm{~m}$ telescope equipped with a $33^{\prime}$ square camera. Over six and half years (2002-2008), this program counted more than 1000 NEA follow-up observations, but only 16 NEA recoveries (Tichá 2009), suggesting that larger (preferably at least $2 \mathrm{~m}$ class) and larger field facilities are needed today for recovery. 
During the past few years, recovery of poorly observed NEAs has become essential to confirm the orbits of oneopposition objects that have not been observed for years since discovery and very short follow-up (typically only a few weeks), some in danger of loss or mispairing with newly discovered NEAs.

Particular attention should be given when telescope time is scarce, requiring a larger aperture, field of view, and mandatory quality control of the astrometry and orbital fitting. Within the European Near Earth Asteroids Research (EURONEAR; Vaduvescu 2008), follow-up and recovery have been the main astrometric tools used for the orbital amelioration of NEAs, potentially hazardous asteroids (PHAs), and virtual impactors (VIs) (Birlan 2010; Vaduvescu 2011, 2013).

Since 2000, A. Milani and his Pisa University team have improved the uncertainty models needed to search for poorly observed asteroids (one-opposition with short arcs, or asteroids that have not been observed for many years), considering nonlinear error propagation models to define the sky uncertainty area, which typically spans an elongated ellipse (Milani 1999a, 2010). It is essential to use these theories to recover one-opposition NEAs, and this could be easily done today using the ephemerides given by the NEODyS server ${ }^{1}$ or the OrbFit Software Package ${ }^{2}$.

When we count the entire NEA database as of Aug. 2017 (about 16500 objects with orbital arcs expressed in days), about $50 \%$ represent one-opposition NEAs (more than 8000 objects), and this percentage is growing because of the accelerated discovery rate of existing and future surveys. A pool of about 400 one-opposition NEAs (5\%) brighter than $V<24$ mag with solar elongation greater than $60^{\circ}$ are recommended for observations at any particular time by the Minor Planet Centre (MPC) at any particular time in their Faint ${ }^{3}$ and Bright ${ }^{4}$ NEA Recovery Opportunities lists. Around opposition, many of these targets escape detection by major surveys because they are faint, because the visibility windows are relatively short, because of fast proper motions, and because of bright Moon and Milky Way interference.

In 2014, we started a pilot recovery program with the aim to observe 100 one-opposition NEAs using the $2.5 \mathrm{~m}$ Isaac Newton Telescope (INT) accessed during at most 30 triggers (maximum $1 \mathrm{~h}$ each available night) through the Spanish TAC ToO time (Target of Opportunity or override mode). This program produced some promising results (about 40 recoveries during only 15 triggers), nevertheless, some visibility windows were lost because telescope access was constrained to only during the allocated Spanish one-third fraction, only when the imaging camera was available, and only during dark time. During the next three semesters, we multiplied the trigger windows by proposing the same program to the other two TACs (UK and Dutch), who have agreed to share the load and granted 15-20 h each during each of the next three semesters, but mostly in "soft" mode (only at the discretion of the observer) and also accepting some twilight time (20 min mostly before morning) so that their own research was not strongly affected. The first semester in 2016 concluded with the last Spanish allocation, and by mid-2016, we reached the goal of recovering more than half of the one-opposition NEAs recommended for observation by the MPC.

\footnotetext{
1 http://newton.dm.unipi $\cdot$ it/neodys/index $\cdot$ php?pc=0

2 http://adams.dm.unipi.it/orbfit

http://www . minorplanetcenter . net/iau/NEO/

FaintRecovery.html

4 http://www .minorplanetcenter.net/iau/NEO/

BrightRecovery.html
}

In this paper we report the achievements of this project, discussing the observing methods and findings, and comparing the INT with other facilities used for similar projects. In Sect. 2 we present the planning tools and observations. The data reduction software and methods are included in Sect. 3, the results are presented in Sect. 4, and we conclude in Sect. 5.

\section{Planning and observations}

Here we present the tools we used for planning, the facilities, and the observing modes.

\subsection{Recovery planning tool}

In April 2010, the "One-opposition NEA Recovery Planning" tool $^{5}$ was written in PHP by Marcel Popescu and Ovidiu Vaduvescu to assist in planning the observations of the one-opposition NEAs retrieved from the Faint and Bright Recovery Opportunities for NEOs MPC lists. The input is the observing night (date) and start hour (UT), the number of steps and time separator (typically $1 \mathrm{~h}$ ), selection of the bright or faint MPC lists, the MPC observatory code, the maximum observable magnitude for the targets, the minimum altitude above horizon, the maximum star density in the field (to avoid the Milky Way), the maximum proper motion, and the maximum positional uncertainty (one sigma) as retrieved by the NEODyS server. The output consists of a few tables (one for each time-step), prioritizing targets based on a few observability factors to choose from, such as the apparent magnitude, altitude, proper motion, sky plane uncertainty, or taking them all into account at once. Other data listed in the output are the stellar density, the angular distance to the Moon, and the Moon altitude and illumination.

\subsection{INT override observations}

The $2.5 \mathrm{~m}$ Isaac Newton Telescope (INT) is owned by the Isaac Newton Group (ING). It is located at $2336 \mathrm{~m}$ altitude at the Roque de los Muchachos Observatory (ORM) on La Palma, Canary Islands, Spain. The mosaic Wide Field Camera (WFC) is located at the $F / 3.3$ INT prime focus, consisting of four CCDs with $2048 \times 409813.5 \mu \mathrm{m}$ pixels each, resulting in a scale of $0.33^{\prime \prime} /$ pixel and a total $34^{\prime}$ square field with a missing small square $12^{\prime}$ in its NW corner. During all runs, we used the Sloan $r$ filter, which suppresses fringing and improves the target signalto-noise ratio $(\mathrm{S} / \mathrm{N})$ in the twilight. The telescope is capable of tracking at differential rates, and we mostly used tracking at half the NEA proper motion in order to obtain a similar measurable trailing effect for both the target and reference stars. The INT median seeing is $1.2^{\prime \prime}$, and we typically required an ORM seeing monitor limit of $1.5^{\prime \prime}$ in order for the triggers to become active.

In Table 1 we include the observing proposals (all three TACs), the number of executed triggers (in bold), and the total granted number of triggers (e.g., 15/30 means that 15 triggers were executed of a maximum allowed 30). Additionally, available fractions during another nine ING discretionary nights ("D-nights") were used to observe a few dozen targets, involving some ING student observers. In total, about 130 INT hours were used for this program. All the observers were invited to become coauthors of this paper.

For each target field, typically 6-8 consecutive images (up to 15 for very faint targets) were acquired with exposures of typically 60-90 s each (up to a maximum $180 \mathrm{~s}$ in a few cases),

\footnotetext{
5 http://www . euronear.org/tools/planningmpc.php
} 
Table 1. Observing proposals and number of triggers activated (in bold) with the Isaac Newton Telescope (INT).

\begin{tabular}{|c|c|c|c|c|c|c|}
\hline Semester & \multicolumn{2}{|l|}{ SP TAC } & \multicolumn{2}{|c|}{ UK TAC } & \multicolumn{2}{|l|}{ NL TAC } \\
\hline $2014 \mathrm{~A}$ & 136-INT09/14A (C136) & $15 / 30$ & & & & \\
\hline 2014B & 088-INT10/14B (C88) & $6 / 20$ & $\mathrm{I} / 2014 \mathrm{~B} / 02(\mathrm{P} 2)$ & $10 / 20$ & & \\
\hline $2015 \mathrm{~A}$ & 033-MULT-2/15A (C33) & $\mathbf{9} / 20$ & $\mathrm{I} / 2015 \mathrm{~A} / 05(\mathrm{P} 5)$ & $1 / 20$ & I15AN003 (N3) & $\mathbf{3} / 20$ \\
\hline $\begin{array}{l}2015 B \\
2016 A\end{array}$ & 001-MULT-2/15B (C1) & $14 / 15$ & $\begin{array}{l}\mathrm{I} / 2015 \mathrm{~B} / 02(\mathrm{P} 2) \\
\mathrm{I} / 2016 \mathrm{~A} / 02(\mathrm{P} 2)\end{array}$ & $\begin{array}{r}11 / 15 \\
6 / 10\end{array}$ & I15BN001 (N1) & $4 / 15$ \\
\hline
\end{tabular}

so that the trail effect would not surpass twice the seeing value. Considering the WFC readout time (49 s in the slow and $29 \mathrm{~s}$ in the fast mode used mostly in this project), one target sequence could take between 10 and $20 \mathrm{~min}$, which means that we could accommodate between three and six targets during a one-hour typical override. For targets with larger uncertainties $\left(3 \sigma \gtrsim 600^{\prime \prime}\right)$, we observed two or three nearby fields that covered more than one degree along the line of variation.

\subsection{Other telescopes}

In addition to the INT override program, three other telescopes accessible to EURONEAR were used to recover a few targets and a few NEA candidate discoveries for a very limited time (about $10 \mathrm{~h}$ in total). The first was the $4.2 \mathrm{~m} \mathrm{~F} / 11$ William Herschel Telescope (WHT) at ORM equipped with the ACAM imaging camera (circular $8^{\prime}$ field) during two D-nights testing and twilight time, and another four nights when the current observer had his targets at very high airmass. The second was the ESA $1 \mathrm{~m} \mathrm{~F} / 4.4$ Observing Ground Station (OGS) equipped with a $45^{\prime}$ square field camera at Tenerife Teide Observatory, used during two nights for the recovery of two target NEAs and to secure three of our NEA incidental discoveries. Additionally, a third telescope was used to follow up a few NEA candidate discoveries, namely the Sierra Nevada Observatory 1.5 m (T150) F/12.5 with the CCDT150 camera $8^{\prime}$ square field.

Table 2 lists the observing log, which includes all the 457 observed fields (437 using the INT, 12 using the WHT, and 4 using the OGS). We ordered this table based on the asteroid designation (first column), then the observing date (start night), listing the apparent magnitude $V$ (according to MPC ephemerides), the proper motion $\mu$ and the positional uncertainty of the targets (as shown on the observing date by MPC at $3 \sigma$ level), the number of acquired images (including nearby fields), and the exposure time (in seconds). In the last three columns we list the current (Jul. 2017) status of the targets (to be discussed next), the MPS publication that includes our recovery, and some comments that can include the PHA classification, other used telescopes (WHT or OGS), the track-and-stack technique (TS, whenever used), and other possible external stations (MPC observatory code) and the date of later recovery (given only for later recoveries when we were unable to find the targets or for joined simultaneous recoveries).

\section{Data reduction}

We present next the data reduction software and quality control methods used to find and measure the targets. Three steps were performed during the day following observations: the image reduction and field correction (by one person), the visual search and measurement of the target and all other moving objects (known or unknown) appearing in each field (distributing the work to a team of a few people), and finally the quality control and reporting of all data to MPC (by the project leader).

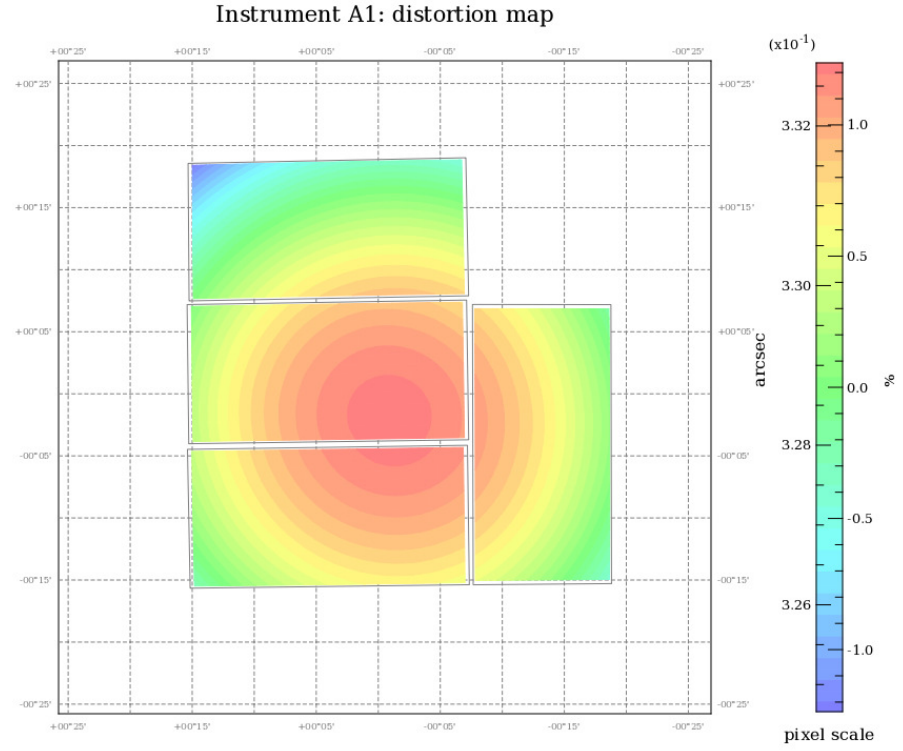

Fig. 1. Typical THELI field distortion of the INT-WFC field.

\subsection{THELI}

Very accurate astrometry (comparable to or lower than the reference star catalog uncertainty, preferably below $0.1^{\prime \prime}$ ) is essential to correctly link and improve the orbits that have been poorly observed in the past, like one-opposition NEAs. Any fast system and prime focus larger field camera (such as INT-WFC) provides quite distorted raw astrometry that needs correction in order to be used for accurate measurements. We used the GUI version ${ }^{6}$ of the THELI software (Erben 2005; Schirmer 2013) to reduce the raw WFC images using the night bias and flat field and to resolve the field correction to all four CCDs in each WFC-observed field by using a third-degree polynomial distortion model. In Fig. 1 we include one typical field distortion map output of THELI (running Scamp), showing pixel scale differences of up to $0.006^{\prime \prime}$ between the center and corners of the WFC field, which can produce errors of up to $40^{\prime \prime}$ when a simple linear astrometric model is applied. For most of the data reduction, we used the PPMXL reference star catalog (Roeser 2010), while UCAC4, SDSS-DR9, or USNO-B1 were used when the field identification failed because of a lack of stars or small dithering between frames.

\subsection{Astrometrica}

The Windows Astrometrica software ${ }^{7}$ is popular among amateur astronomers for field registering, object identification, and astrometric measurement of the asteroids; it is written by the Austrian

\footnotetext{
6 https://www .astro.uni-bonn.de/theli/gui/index.html

7 http://www.astrometrica.at
} 


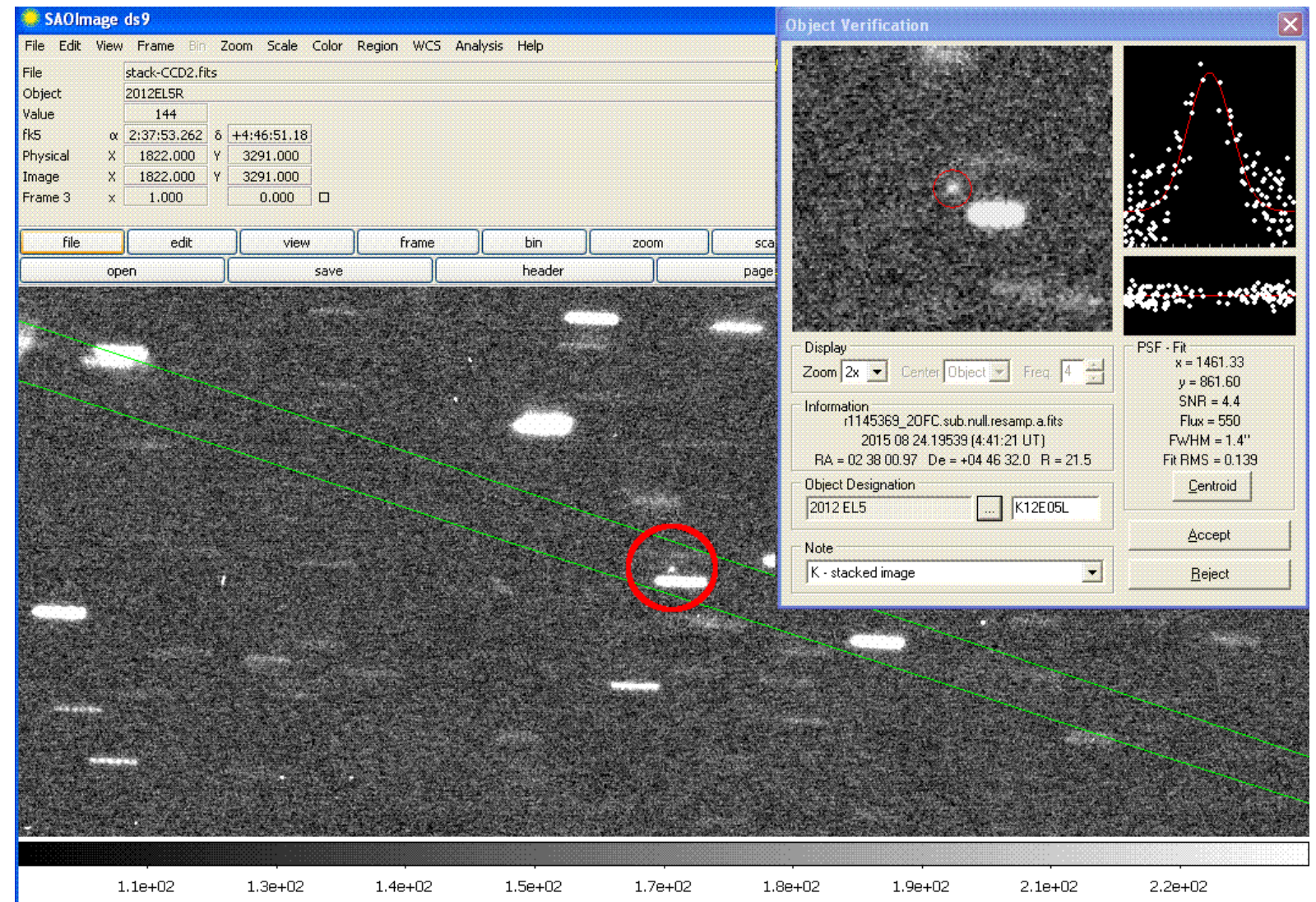

Fig. 2. Track-and-stack Astrometrica image (composition of six individual images using the "add" option) overlaid on DS9 the NEODyS uncertainty ellipse (green) that we used to find the target NEA (2012 EL5, circled in red).

amateur astronomer Herbert Raab. We used it after every run, updating the MPCORB database to take all newly discovered asteroids and updated orbits into account. In 2014, Ruxandra Toma and Ovidiu Vaduvescu wrote a user guide manual ${ }^{8}$ (21 pages) aimed for training the new members of the reduction team.

\subsubsection{Classic blink search}

We used Astrometrica for each observed WFC field to independently blink the four CCDs, identifying all moving sources (as known or unknown asteroids), and measuring them. Typically, between 1 and $2 \mathrm{~h}$ were spent by one reducer for each WFC field. Although Astrometrica is capable of automatic identification of moving sources, given the faintness of our NEA targets, we decided to use visual blink and manual measurements. In addition to the targeted NEA, typically up to a few dozen main-belt asteroids (MBAs, about half of them known and half unknown) could be identified in good seeing conditions in each observed WFC field.

\subsubsection{Track-and-stack}

When the NEA target could not be seen using the classic blink search, then the Astrometrica track-and-stack method ("TS") was used, either with the "median" option to eliminate most of the stars, or with the "add" option to improve the detection of extremely faint targets $(S / N=2-3)$. The linear apparent motion assumed by the TS procedure could be affected by the diurnal

\footnotetext{
8 http://www. euronear.org/manuals/ Astrometrica-UsersGuide-EURONEAR.pdf
}

paralax effect, and the TS detection could fail during very close flybys or/and a longer observing time that was affected by diurnal effects, but we consider that none of our targets was affected by these circumstances, as the length of each observing sequence was short. To limit the search area, we developed a method using DS9 to load the Astrometrica TS image and overlay the NEODyS $3 \sigma$ uncertainty, thus restraining the visual search to a very thin ellipse area (possible to save and load as a DS9 region) typically passing across the central CCD4 (holding the target most of the time) or/and nearby CCDs or fields. We include in Fig. 2 one typical DS9 overlay (NEA 2012 EL5 on 23 Aug. 2015 with uncertainty $3 \sigma=788^{\prime \prime}$ prolonging to the nearby CCD2), which allowed the identification of the target falling exactly on the major axis of the NEODyS uncertainty ellipse overlaid on the stack of $6 \times 60 \mathrm{~s}$ individual images.

\subsection{Quality control}

Astrometrica can easily identify moving sources with wellknown asteroids (observed at two oppositions at least) by calculating their ephemerides using an osculation orbit model with orbital elements very close to the observing time, which provides a very good accuracy of $\sim 1^{\prime \prime}$. After each observing night, we used Astrometrica to check all moving sources that were visible in each WFC field against known MBAs included in the updated MPCORB database ${ }^{9}$. Nevertheless, one-opposition objects and especially NEAs closer to Earth are affected by positional uncertainties, and they should be checked using additional tools. 


\subsubsection{AstroCheck, FITSBLINK, and O-C calculator}

In 2015, Lucian Hudin developed the EURONEAR PHP tool AstroCheck ${ }^{10}$ to verify the consistency of all astrometric measurements obtained in each WFC field (known or unknown asteroids). This tool assumes that a simple linear regression model holds for relatively short and contiguous observational arcs like those observed during 10-20 min runs for each target of our recovery project. A maximum error (default $0.3^{\prime \prime}$ consistent with WFC pixel size) is allowed, all other outliers being flagged in red, these positions being revised or discarded by the reducer.

We used the server FITSBLINK ${ }^{11}$, which identifies known objects and provides tables and plots to check the $\mathrm{O}-\mathrm{C}$ (observed minus calculated) residuals for all asteroids (mostly MBAs) identified by Astrometrica in each WFC field. The calculation of the asteroid positions is based on osculating elements near the current running date, so the identification is correct for checks after each observing run, but it could fail for older measurements. The great majority of the residuals are scattered around the origin in the $\alpha-\delta$ FITSBLINK plots, proving the correct identification of the MBAs. Some asteroids (MBAs and target NEAs) show normal non-systematic clustering around values different than zero (typically by a few arcseconds), suggesting the correct identification of poorer known orbits. If any object presents systematic $\mathrm{O}-\mathrm{C}$ residuals (typically located far from the origin), then this most probably represents an erroneous identification, and FITSBLINK flags these objects as unknown.

In addition to FITSBLINK, to check MBAs residuals, we used the EURONEAR tool $\mathrm{O}-\mathrm{C}$ Calculator ${ }^{12}$, which provides tables to check for accurate residuals for each target NEA. The residuals are calculated based on accurate ephemerides run using the OrbFit planetary perturbation model that is automatically queried via NEODyS ${ }^{13}$. Each correctly identified one-opposition NEA target must show normal non-systematic scatter (located around a center different than the origin), otherwise the identification is false.

For the target NEAs, the FITSBLINK and the O-C Calculator residuals could be randomly spread (non-systematic) around a point which may be different than the origin, while for most MBAs, the $\mathrm{O}-\mathrm{C}$ values are typically spread around the origin.

\subsubsection{Find_Orb and orbital fit}

The Find_Orb software ${ }^{14}$ is a user-friendly popular orbit determination software under Windows or Linux for fitting orbits of solar system objects based on existing observations, written by the US American amateur astronomer Bill Gray. We used Find_Orb to finally check NEA targets that showed larger positional uncertainties. Past observations were downloaded from the MPC Orbits/Observations database ${ }^{15}$, which was updated with our proposed identification and astrometric measurements, before using Find_Orb in two steps.

First, using only past positions, an orbit is fit in a few (typically 3-4) converging steps by activating all perturbers and rejecting outlier measurements greater than $1^{\prime \prime}$ in $\alpha$ or $\delta$. Virtually all fits should produce an overall $\sigma$ root-mean-square deviation smaller than $1^{\prime \prime}$. Second, we append our measurements to the input observation file to load in Find_Orb to attempt an improved

\footnotetext{
10 http://wWW . euronear .org/tools/astchk.php

11 http://www. fitsblink.net/residuals

12 http://www . euronear .org/tools/omc.php

13 http://newton.dm. unipi.it/neodys

14 https://www.projectpluto.com/find_orb.htm

15 http://www.minorplanetcenter.net/db_search
}

orbital fit in a few (3-4) converging steps, which must conserve or slightly improve $\sigma$ (typically by $0.01-0.02^{\prime \prime}$ ) and show random distributions in both $\alpha$ and $\delta$ (typically below $0.3^{\prime \prime}$ in module) around zero for our measurements.

If any target presents a systematic $\mathrm{O}-\mathrm{C}$ trend or increases the $\sigma$ orbital fit, then the identification is false or the candidate (typically very faint or found using the TS technique) represents an artifact and is discarded.

\section{Results}

\subsection{Targeted NEAs}

We accessed time for the NEA recovery program during 102 nights: 94 nights using the INT (mostly in override mode for a maximum of $1 \mathrm{~h}$ each night and using some D-nights), plus another 6 nights using the WHT and 2 nights using the OGS. We targeted 368 one-opposition NEAs (including 56 PHAs), observing 453 fields: 437 with the INT (representing $96 \%$ of the program), 12 with the WHT, and 4 fields with the OGS. We recovered 290 NEAs in total (79\% from all 368 targets), of which 280 targets were recovered with the INT. One hundred and three recovered objects (representing 28\% of all targets) were observed at second opposition only by EURONEAR, proving the importance of planned recovery compared with shallower surveys.

Orbital arcs were typically prolonged from a few weeks to a few years, our oldest one-opposition recoveries improving orbits of objects that were not seen for up to 16 years (1999 DB2 and 1999 JO6). Based on Table 2, the user can evaluate the extended arc (in years) by simply subtracting the discovery year (first four digits in the first column NEA designation) from the observing date (first four digits standing for the year), the oldest recoveries being included in the first part of the table.

Because they were not recovered during the first attempt, 67 NEAs (18\%) were targeted multiple (typically two to three) times, some of them even up to six times $(2008 \mathrm{ON}$, resolved during four nights), in order to secure recoveries of very faint objects that were seen only with TS and to minimize the risk of false detections.

We sorted our findings into a few groups that we list in Table 2 under the Status column:

- REC - recovery (followed by other stations);

- RECO - recovery only (not followed by others);

- RECJ - recovery joined (simultaneously with others);

- RECR - revised recovery (in 2017 or following other later recovery);

- NOTF - not found (but found by others later);

- NOTFY - not found yet (by any other station).

We were unable to find 79 objects (21\% of all 368 targets) that are marked with status NOTF or NOTFY in Table 2 for several reasons, the most common being that some targets were fainter than originally predicted, others were affected by cirrus, calima, or late twilight, and a few were hidden by bright stars or have fallen in the WFC gaps. Of these, 46 objects (12\%) were recovered later by other programs or surveys (status NOTF), and another 33 objects (9\%) have not been found yet (by July 2017); these are marked with the status NOTFY. Additionally, we were able to recover 16 objects later (status RECR), following a revised search (carried out in 2017) based on an orbit that was improved by other programs. Here we report the most efficient programs (MPC code, facility, and number of later recoveries 


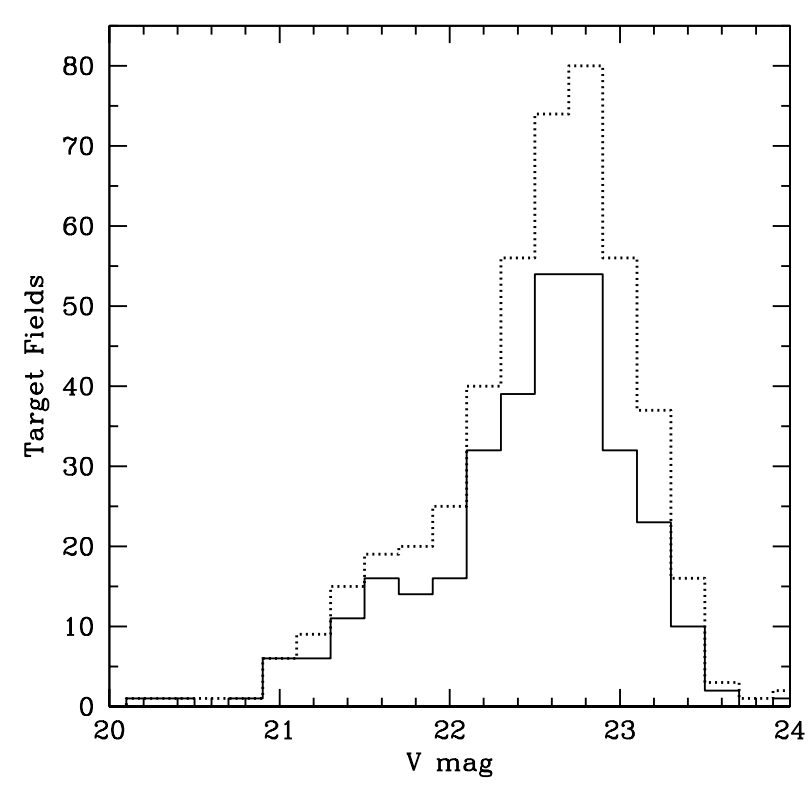

Fig. 3. Distribution of the NEA apparent magnitude.

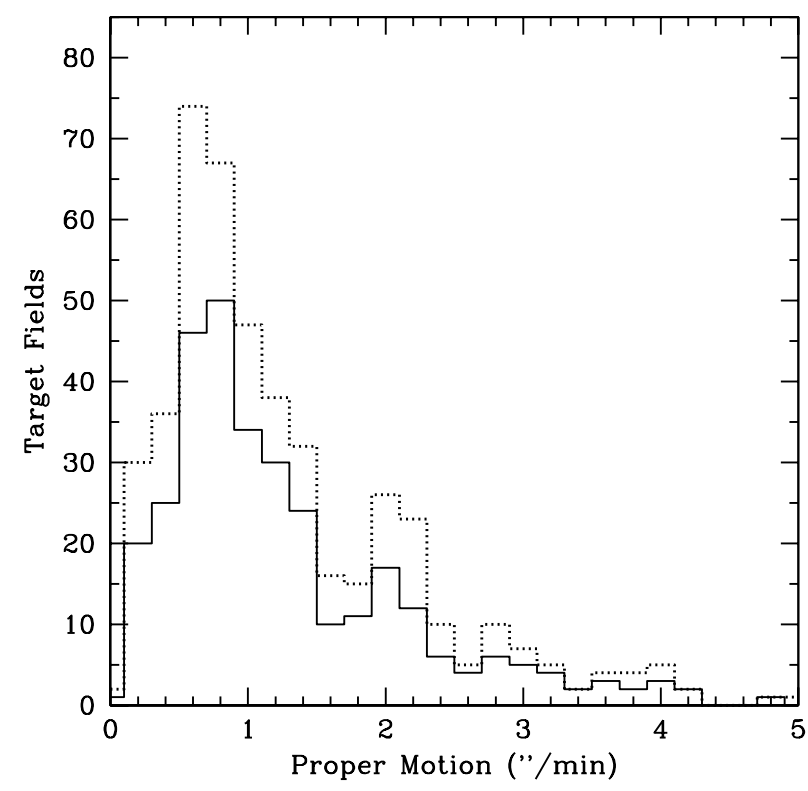

Fig. 4. Distribution of the NEA proper motion.

missed by us): 568+T12 (CFHT and UH telescopes, 28 recoveries or $7 \%$ of all our targets), 926 (Tenagra II, 9 recoveries), J04 (ESA/OGS Tenerife, 8), F51 (Pan-STARRS 1, 6), 807+W84 (CTIO and Blanco/DECam, 5), 291 (Spacewatch II, 4), H21 (ARO Westfield, 4), 705 (SDSS, 3), G96 (Catalina, 2), 695 (KPNO, 2), 033 (KSO, 2), H36 (Sandlot 2), 675+I41 (Palomar and PTF, 2), 309 (Paranal VLT, 1), G45 (SST Atom Site, 1), and T08 (ATLAS-MLO, one recovery).

In Fig. 3 we present the magnitude distribution of all targeted fields (plotted with a dotted line) and recovered targets (solid line). Most targets had $V \sim 22.8$, and most targets were also recovered around $V \sim 22.8$. A few fainter objects were targeted and some were recovered close to $V \sim 24.0$ using the TS technique.

In Fig. 4 we present the proper motion distribution of all targeted fields (dotted line) and recovered targets (solid line). Most targets had relatively small proper motion (around $\mu \sim 0.7^{\prime \prime} / \mathrm{min}$, sampling the morning small solar elongation targets), while

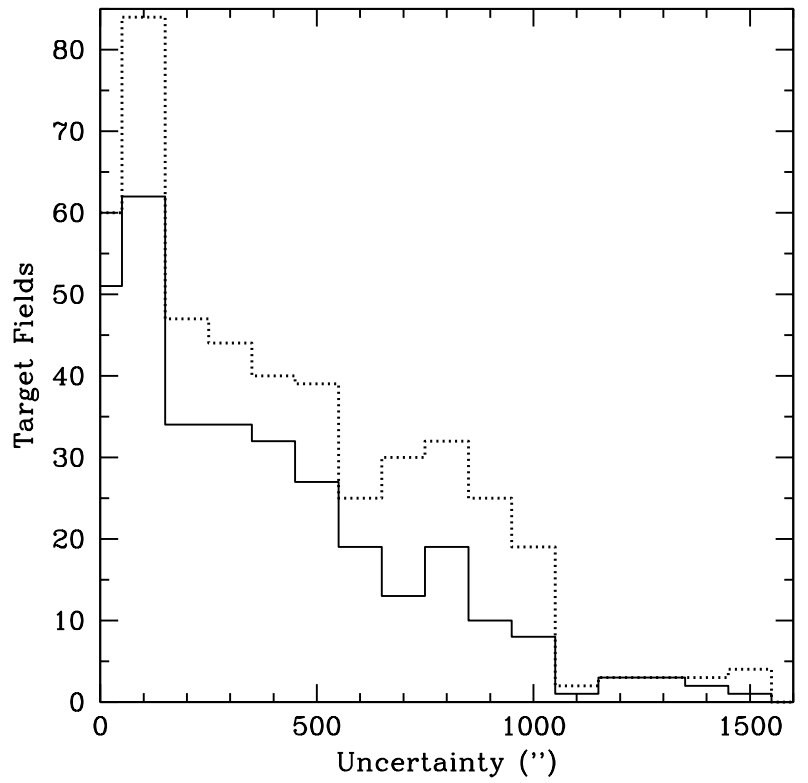

Fig. 5. Distribution of the NEA $3 \sigma$ uncertainty.

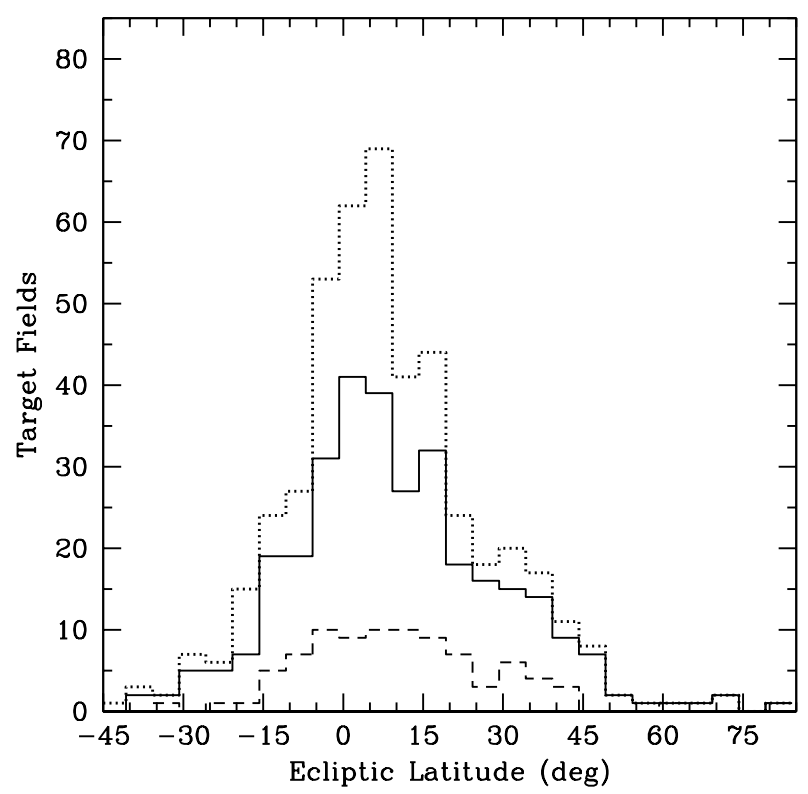

Fig. 6. Distribution of the NEA ecliptic latitudes.

another small peak is visible around $\mu \sim 2.0^{\prime \prime} / \mathrm{min}$ and other faster objects (up to $\mu \sim 5.0^{\prime \prime} / \mathrm{min}$ ) sample closer flybys and opposition apparitions.

In Fig. 5 we present the $3 \sigma$ positional uncertainty distribution of all targeted objects (dotted line) and recovered targets (solid line). Most targets had $3 \sigma<1000^{\prime \prime}$ (due to the selection limit), and there were 20 targets with uncertainties of up to $3000^{\prime \prime}$ (outside the plot) for which we observed two or three nearby fields.

Figure 6 plots the histogram counting all the observed fields (upper dotted line) as a function of the ecliptic latitude $(\beta)$, showing that most fields were observed between $-20^{\circ}<\beta<+50^{\circ}$. The recovered targets are plotted with a continuous line (in the middle), and the one-night recoveries are plotted with a dashed line (in the bottom). They are discussed in Sect. 4.2.

Figure 7 plots the $\mathrm{O}-\mathrm{C}$ residuals (observed minus calculated) for 1854 NEA measurements from the NEODyS database 


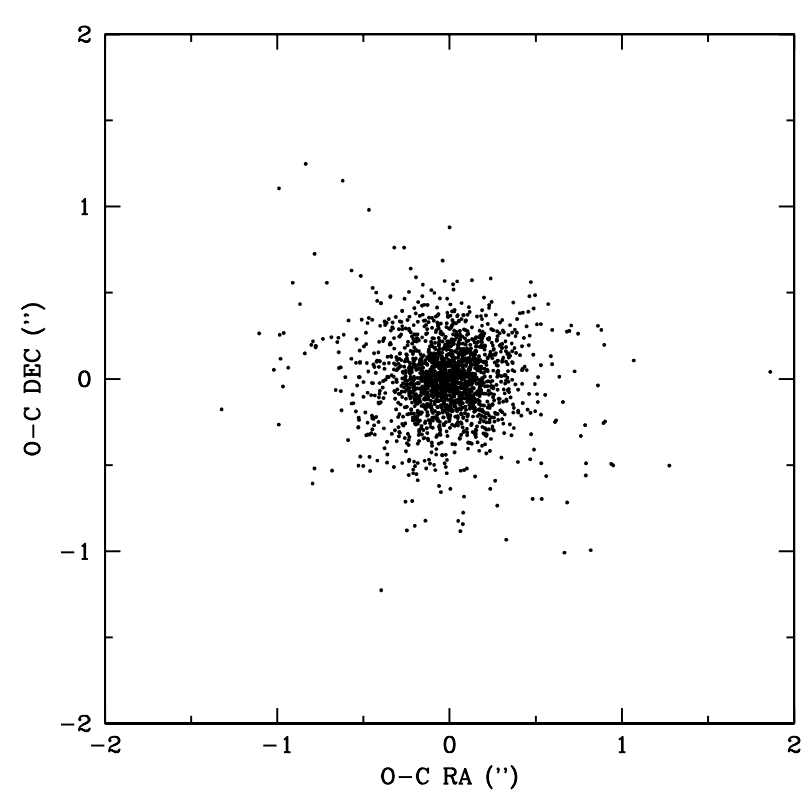

Fig. 7. O-C residuals for 1854 positions of 280 one-opposition NEAs.

based on the improved orbits (by 3 Aug. 2017). Most of the points are located around the origin, with a standard deviation of $0.26^{\prime \prime}$ in $\alpha$ and $0.34^{\prime \prime}$ in $\delta$. Only eight points ( $0.4 \%$ of all data) sit outside $1^{\prime \prime}$ in either $\alpha$ or $\delta$; they represent measurements of very faint targets.

\subsection{Main-belt asteroids and the NEA misidentification risk}

All moving sources found through blinking in the WFC images were identified with known asteroids or were labeled as unknown asteroids and reported to MPC promptly after each run (typically during the next day). By checking the MPCAT-OBS and the ITF archives ${ }^{16}$, we were able to count about $22000 \mathrm{ob}-$ servations of about 3500 known minor planets (mostly MBAs) and about 10000 observations of about 1500 unknown objects (most consistent with MBAs) reported by our team between Sep 2013 and Oct 2016 as part of this project.

In a series of papers, A. Milani and colleagues proposed new algorithms to better approximate and predict the recovery region of poorly observed asteroids and comets by using a nonlinear theory to compute confidence boundaries on the modified target plane (Milani 1999a,b, 2000, 2001). This theory was implemented in NEODyS, which has been used by us to plot the uncertainty regions of the one-opposition NEA targets, which is essential for a correct identification of very faint asteroids (found with the TS technique) and one-night recoveries. We have made 91 one-night recoveries (counted by Aug. 2017), meaning that targets were identified and measured during only one night as part of our NEA recovery project (neither by us during another night, nor by others until Aug. 2017). There is some risk for misidentification in these cases when some targets fall in a dense ecliptic field populated with MBAs. To assess this risk, in Fig. 6 we show the ecliptic latitude distribution by plotting all target fields (upper dotted line), the recovered target fields (middle solid line), and one-night recoveries (bottom dashed line). When we counted the recoveries close to the ecliptic $\left(-5^{\circ}<\beta<+5^{\circ}\right)$, we found 19 risk cases ( $20 \%$ of all one-night recoveries) when target NEAs might be confused with MBAs moving at similar direction

\footnotetext{
${ }^{16}$ http://WWW . minorplanetcenter . net/iau/ECS/MPCAT-OBS/ MPCAT-OBS.html
}

and rate. The following five precaution measures (adopted for most observed fields) minimize false detections in these cases:

- We detected all known moving objects and identified all know MBAs and other possible known NEAs in all fields.

- We ensured that $\mathrm{O}-\mathrm{Cs}$ for the NEA candidate detections were non-systematic (they might spread around a point different than zero, but should not show any systematic trend).

- We plotted the predicted NEODyS uncertainty regions for the target NEAs, ensuring that each candidate NEA detection falls very close to (typically within $1^{\prime \prime}$ ) the long axis of the NEODyS uncertainty ellipse.

- We fit each candidate detection (positions) to the existing orbit, downloading old observations from the MPC database and using Find_Orb to fit the improved orbit, ensuring that the orbital rms remains the same or improves slightly (typically by $\left.0.01-0.02^{\prime \prime}\right)$ after the fit and that our candidate positions $\mathrm{O}-\mathrm{Cs}$ are non-systematic and spread around zero in the new orbital fit.

- We ensured that our measured magnitudes of all targets were similar to their predicted magnitudes (typically within 1 mag, allowing for the unknown color index $r-V$, for some errors in the magnitudes, and for a higher amplitude light-curve that might be due to more elongated objects).

Using all these checks, we reduced the risk to confuse any onenight target NEA with other MBAs. This is supported by many other one-night recoveries that were confirmed later by other stations (marked with REC or RECJ in Table 2).

\subsection{New serendipitous INT NEA discoveries}

Vaduvescu (2015) reported the first EURONEAR NEA discoveries from La Palma that were serendipitously found as unknown fast-moving objects in some INT WFC fields taken in 2014 as part of the present one-opposition NEA recovery project. Here we present discovery circumstances of four other secured NEAs in 2015, plus two other probably lost NEOs, together with their composite images shown in Fig. 8. In total, EURONEAR discovered and secured nine NEAs in 2014 and 2015, the only such findings from La Palma and using the INT.

\subsubsection{HA117}

The very fast $15^{\prime \prime} / \mathrm{min}$ and relatively faint $R \sim 22 \mathrm{mag}$ NEA candidate EUHI640 was discovered by Lucian Hudin on 23/24 Apr. 2015 in the one-opposition WFC field of NEA 2003 WU153 observed by Matteo Monelli and Lara Monteagudo (MPS 603500). Thanks to the INT override access, the object was recovered the next night by the same team, who scanned 25 WFC fields spanning the MPC uncertainty area, then by the INT, and four days later, it was caught by the VLT close to the South celestial pole (MPS 604697). It became 2015 HA117, estimated at $H=27.2$ and with a size of 10-24 m, apparently having an Amor orbit with MOID $=0.01832$ a.u. (based on a seven-day arc), and has remained unobserved since then.

\subsubsection{LT24}

The fast 8 " $/$ min EUVI053 $R \sim 21.3$ mag NEA candidate was discovered by Victor Inceu in images taken on 14/15 Jun. 2015 by Stylianos Pyrzas, who chased the known one-opposition 2012 HO2 NEA (MPS 611632). It was saved during the next night with the INT by the same team, then followed-up by 


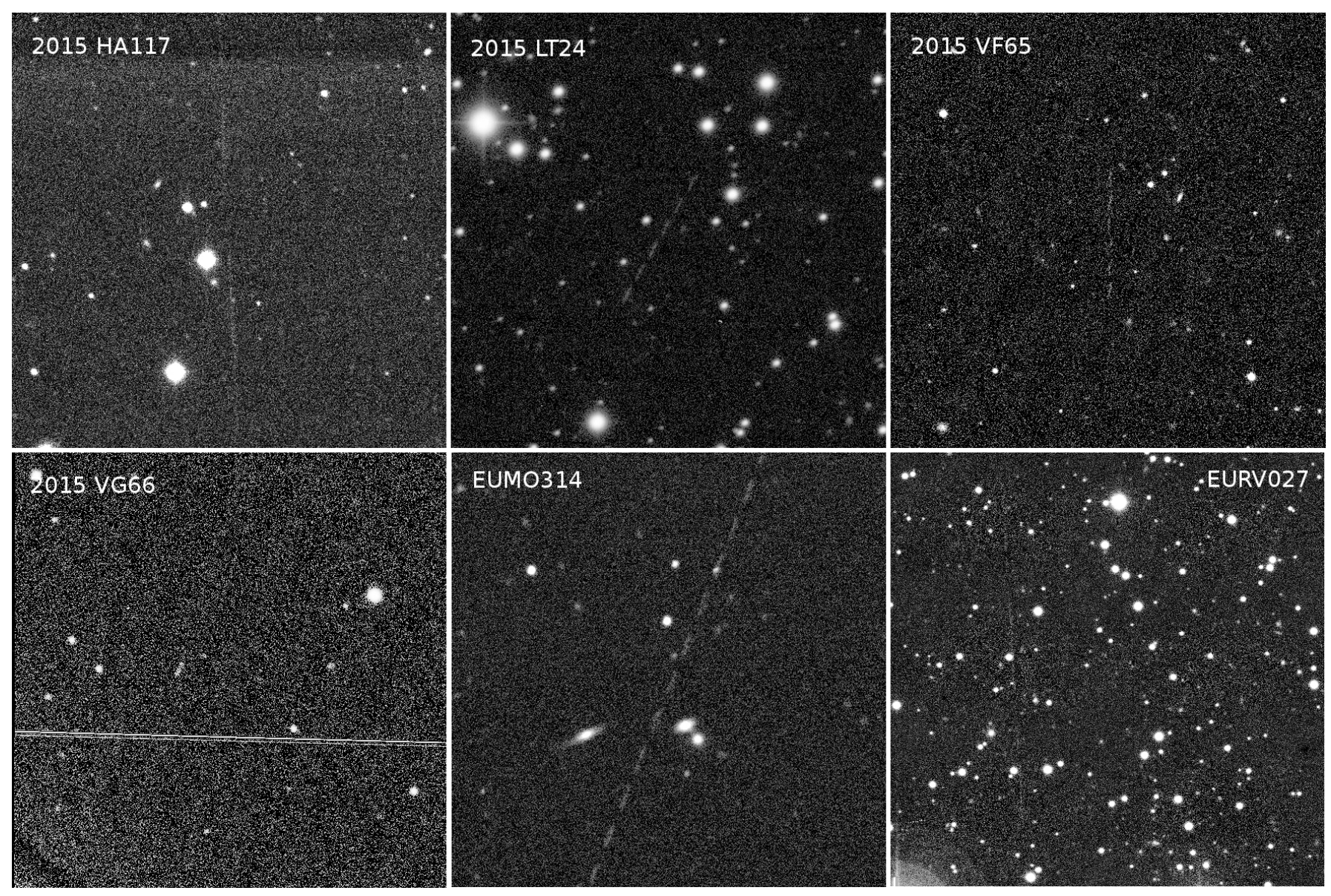

Fig. 8. Composite images of the six serendipitous NEA discoveries (four secured and two lost objects) by EURONEAR using the INT in 2015. Crops are in normal sky orientation (north is up, east to the left), $3^{\prime} \times 3^{\prime}$ field of view, except for EURV027, which is barely visible as three very long vertical trails at the left of the $6^{\prime} \times 6^{\prime}$ field.

other telescopes related to EURONEAR (OGS $1 \mathrm{~m}$ and Sierra Nevada $1.5 \mathrm{~m}$ ), which prolonged its arc to 11 days. Designated as 2015 LT24, it is a relatively large $H=22.4100-220 \mathrm{~m}$ Apollo object with MOID $=0.15616$ a.u.

\subsubsection{VF65}

EUHV001 was another very fast $\left(11^{\prime \prime} / \mathrm{min}\right) R \sim 22$ mag NEA candidate discovered as a trailing object by Lucian Hudin on 7/8 Nov. 2015, searching for the one-opposition target 2010 VC72 (right field) observed by Odette Toloza (MPS 645818). Thanks to the NEOCP posting, it was saved on next night by Spacewatch and the OGS $1 \mathrm{~m}$ and became 2015 VF65, which was followedup with the INT and another station later (13 day arc). This resolved into an Apollo orbit with an MOID $=0.05225$ a.u. and $H=26.1$, corresponding to a size of $18-40 \mathrm{~m}$.

\subsubsection{VG66}

EUHV002 was a moderate NEA candidate $\left(\mu=1.6^{\prime \prime} / \mathrm{min}\right)$ relatively bright $R=19.4 \mathrm{mag}$, first seen on $8 / 9$ Nov. 2015 by our most prolific discoverer Lucian Hudin in one of the 15 chasing fields (EUHV001I) that were taken by Odette Toloza to secure our previous NEA candidate (MPS 645822). Despite its relatively modest MPC NEO score (42\%), we decided to chase it because of its location above the NEA border on the $\epsilon-\mu$ plot (Vaduvescu 2011). On the next night, it was secured by the INT observers Odette Toloza and Christopher Manser, then precovered in Pan-STARRS images by Peter Veres (priv. comm.), and later observed by other stations (18-day arc). It has an Apollo orbit with an MOID $=0.01991$ a.u. and $H=23.2$, corresponding to a quite large object of $72-161 \mathrm{~m}$.

\subsubsection{EUMO314}

This very fast NEA candidate $\left(\mu=15^{\prime \prime} / \mathrm{min}, R \sim 19.3 \mathrm{mag}\right)$ was seen by Teo Mocnik in 15 images taken on 1/2 Mar. 2015 by Fatima Lopez while chasing another faint NEA candidate (EUMO311). It was lost, unfortunately, the WFC being replaced on next morning by the IDS spectrograph, while no other station could save it.

\subsubsection{EURV027}

This extremely fast NEO candidate $\left(\mu=40^{\prime \prime} / \mathrm{min}\right)$ was seen by Ovidiu Vaduvescu as four very faint (probably $R \sim 23 \mathrm{mag}$ ) and long trails in images taken on 14/15 Aug. 2015 by Joan Font in the 2013 VM4 target field. This should correspond either to a very small (a few meter) object close to opposition or more likely a tiny geocentric object (Gareth Williams, priv. comm.). It is barely visible in Fig. 8 as three vertical very long trails on the left side of the composite image. 


\subsubsection{Other NEA candidates}

About 15 other slower $\left(\mu<1.5^{\prime \prime} / \mathrm{min}\right)$ and sometimes extremely faint $(S / N<5)$ NEA candidates were found in some other fields scanned by our program. Most of them were chased with the INT on the next nights, and we posted some on the NEOCP list. Many of them could not be recovered (even going deeper with the INT), suggesting that they are artifacts, while others were recovered and were found to be MBAs or close NEA species. We note the following: EUHV056 - a probable Jupiter Trojan (65\%, according to MPC), 2014 RC13 (EUMO201) - Jupiter Trojan (MPO 311499), 2015 QT4 (EURV028) - Hungaria (MPO 382801), and 2014 LP9 (EUHT164) - Mars crosser (MPO 300699).

\section{Conclusions}

A project for recovering one-opposition NEAs recommended by the MPC was carried out during a fraction of 102 nights ( $\sim 130 \mathrm{~h}$ total) between 2013 and 2016 using the INT telescope equipped with the WFC camera. We accessed this time as part of ten proposals with time awarded by three committees mostly in soft-override mode and accepting some twilight time, plus other available time during a few D-nights. The data were rapidly reduced (typically during the next day) by a core team of about ten amateurs and students led by the PI, who checked and promptly reported all data to the MPC. We outline the following achievements:

- We targeted 368 one-opposition NEAs (including 56 PHAs) for which we observed 437 WFC fields with the INT.

- We recovered 290 NEAs (79\% from all targets), sorted into four groups (REC, RECO, RECJ, and RECR), the majority with the INT (280 targets).

- Most targets and recovered objects have magnitudes centered around $V \sim 22.8$ mag (typically recovered through blink), while some are as faint as $V \sim 24$ mag (only visible with track-and-stack and search in the uncertainty ellipse).

- One hundred and three objects (28\% of all targets) have been recovered only by EURONEAR (but no other survey, until Aug. 2017 at least).

- Orbital arcs were prolonged typically from a few weeks to a few years, our oldest recoveries improving orbits of objects that have not been seen for up to 16 years.

- Sixty-seven NEAs (18\%) could not be found during a first attempt, and they were targeted multiple (typically two to three) times.

- Forty-six objects (12\% of all targets) were not found, but were recovered later by other programs or surveys (UH+CFHT 7\%, Tenagra, ESA OGS, and major surveys less than $2 \%$ of our targets each).

- Most targets were slow $\left(\mu \sim 0.7^{\prime \prime} /\right.$ min sampling the morning small solar elongation targets), others concentrated around $\mu \sim 2.0^{\prime \prime} / \mathrm{min}$, while others are faster (up to $\mu=5.0^{\prime \prime} / \mathrm{min}$ ).

- Given the WFC $34^{\prime}$ field, our selection limit in positional uncertainty was $3 \sigma<1000^{\prime \prime}$, but we allowed 20 targets with uncertainties up to $3 \sigma=3000^{\prime \prime}$ for which we observed two or three nearby fields.

- The O-C residuals for 1854 NEA measurements show that most measurements are located closely around the origin, with a standard deviation $0.26^{\prime \prime}$ in $\alpha$ and $0.34^{\prime \prime}$ in $\delta$.

- We identified 22000 observations of about 3500 known minor planets (mostly MBAs) and about 10000 observations of about 1500 unknown objects (most consistent with MBAs), which were measured and reported to the MPC by our team.
- Four new NEAs were discovered serendipitously in the analyzed fields and were then secured with the INT and other telescopes, while two more NEAs were lost due to very fast motion and lack of rapid follow-up time. Nine designated NEAs are discovered by the EURONEAR in 2014 and 2015.

- Three hundred fifteen MPS publications, including data for one-opposition NEAs, were recovered during this project.

Acknowledgements. The PI of this project is indebted to the three TACs (Spanish, British, and Dutch) for granting INT time (ten proposals during five years) in soft-override mode, which was essential to complete this project and secure most discoveries. Special thanks are due to M. Micheli (ESA-SSA), observers P. Ruiz, D. Abreu, and the other TOTAS team (D. Koschny, M. Busch, A. Knöfel, E. Schwab) for the ESA OGS $1 \mathrm{~m}$ follow-up of 2015 LT24, 2015 VF65, and the attempt to observe 2015 VG66. Acknowledgements are due to R. Duffard and S. Martin Ruiz (IAA Granada) for granting some time at the Sierra Nevada Observatory (EURONEAR node) with their $1.5 \mathrm{~m}$ telescope to secure 2015 LT24 and 2015 VG66. Many thanks to O. Hainaut (ESO) and M. Micheli (ESA) for the VLT astrometry of 2015 HA117 (extremely fast, faint, and close to the South Pole in just a few days), which prolonged its orbit to a seven-day arc. I.O. acknowledges support from the European Research Council (ERC) in the form of Advanced Grant, cosmicisM. R.T. acknowledges funding for her La Palma trip to Armagh Observatory, which is core-funded by the Northern Ireland Government. The research led by BTG, CJM, and NPGF has received funding from the European Research Council under the European Union's Seventh Framework Programme (FP/2007-2013) / ERC Grant Agreement No. 320964 (WDTRACER). Thanks are due to the anonymous referee, whose suggestions helped us to improve the paper.

\section{References}

Birlan, M., Vaduvescu, O., Tudorica, A., et al. 2010, A\&A, 511, A40 Boattini, A., \& Forti, G. 2000, Planet. Space Sci., 48, 939

Erben, T., Schirmer, M., Dietrich, J. P., et al. 2005, Astron. Nachr., 326, 432 Milani, A. 1999a, Icarus, 137, 269

Milani, A. 1999b, Icarus, 140, 408

Milani, A. 2000, Icarus, 144, 39

Milani, A. 2001, Icarus, 151, 150

Milani, A., \& Gronchi, G. F. 2010, Theory of Orbit Determination (Cambridge, UK: Cambridge University Press)

Roeser, S., Demleitner, M., \& Schilbach, E. 2010, AJ, 139, 6, 2440

Schirmer, M. 2013, ApJS, 200, 21

Tatum, J., Balam, D., \& Aikman, G. C. L. 1994, Planet. Space Sci., 42, 611

Tichá, J., Tichý, M., \& Moravec, Z. 2000, Planet. Space Sci., 48, 955

Tichá, J., Tichý, M., \& Kocer, M. 2002, Icarus, 159, 351

Tichá, J., Tichy, M., Kocer, M., \& Honkova, M. 2009, Meteor. Planet. Sci., 44, 1889

Vaduvescu, O., Birlan, M., Colas, F., Sonka, A., \& Nedelcu, A. 2008, Planet. Space Sci., 56, 1913

Vaduvescu, O., Birlan, M., \& Tudorica, A. 2011, Planet. Space Sci., 59, 1632 Vaduvescu, O., Birlan, M., \& Tudorica, A. 2013, Planet. Space Sci., 85, 299 Vaduvescu, O., Hudin, L., \& Tudor, V. 2015, MNRAS, 449, 1614

${ }^{1}$ Isaac Newton Group of Telescopes (ING), Apto. 321, 38700 Santa Cruz de la Palma, Canary Islands, Spain

e-mail: ovidiu.vaduvescu@gmail.com

2 Instituto de Astrofísica de Canarias (IAC), C/vía Láctea s/n, 38205 La Laguna, Tenerife, Spain

3 Departamento de Astrofísica, Universidad de La Laguna, 38206 La Laguna, Tenerife, Spain

4 Amateur Astronomer, ROASTERR-1 Observatory, 400645 Cluj Napoca, Romania

5 Unidad de Astronomía, Facultad Ciencias Básicas, Universidad de Antofagasta, Chile

6 Astronomical Institute of the Romanian Academy, 5 Cutitul de Argint, 040557 Bucharest, Romania

7 Dpto. de Física Aplicada I, Escuela de Ingeniería de Bilbao, Universidad del País Vasco, 48940 Bilbao, Spain

${ }^{8}$ National Solar Observatory, 3665 Discovery Drive, Boulder, CO 80303, USA

9 Romanian Society for Meteors and Astronomy (SARM), Str. Tineretului 1, 130029 Targoviste, Romania 
10 Amateur astronomer, 438 Cluj Napoca, Romania

11 Bucharest Astroclub, B-dul Lascar Catargiu 21, sect 1, Bucharest 010662, Romania

12 Institut de Mécanique Céleste et de Calcul des Éphémérides (IMCCE) CNRS - UMR 8028, Observatoire de Paris, 77 avenue Denfert-Rochereau, 75014 Paris Cedex, France

13 Amateur astronomer, Schela Observatory, 800259 Schela, Romania

14 Faculty of Sciences, University of Craiova, Str. Alexandru Ioan Cuza 13, 200585 Craiova, Romania

15 Horia Hulubei National Institute for R\&D in Physics and Nuclear Engineering (IFIN-HH), Str. Reactorului 30, Magurele, Romania

16 Armagh Observatory and Planetarium, College Hill, Armagh BT61 9DG, UK

17 Instituto de Astrofísica e Ciências do Espaço, Universidade do Porto, CAUP, Rua das Estrelas, 4150-762 Porto, Portugal

18 Nicolaus Copernicus Astronomical Center, Bartycka 18, 00-716 Warsaw, Poland

19 Instituto de Astrofísica de Andalucía (IAA-CSIC), Glorieta de la Astronomía, S/N, 18008 Granada, Spain

20 Institute for Astronomy, University of Edinburgh, Royal Observatory, Blackford Hill, Edinburgh EH9 3HJ, UK

21 European Southern Observatory, Karl-Schwarzschild-Str. 2, 85748 Garching, Germany

22 Qatar Environment and Energy Research Institute (QEERI), HBKU, Qatar Foundation, PO Box 5825, Doha, Qatar

23 Laboratoire d'Astrophysique de Bordeaux, Univ. Bordeaux, CNRS, B18N, allée Geoffroy Saint-Hilaire, 33615 Pessac, France

24 Centro de Astrobiología (INTA-CSIC), Dpto. de Astrofísica, ESAC Campus, Camino bajo del Castillo s/n, 28692 Villanueva de la Cañada, Madrid, Spain

25 Centre for Astrophysics Research, Science and Technology Research Institute, University of Hertfordshire, College Lane, Hatfield AL10 9AB, UK

26 Department of Physics, University of Warwick, Coventry CV4 7AL, UK
27 Department of Physics and Astronomy, University of Sheffield, Sheffield S3 7RH, UK

28 Astrophysics Research Centre, School of Mathematics and Physics, Queen's University, Belfast BT7 1NN, UK

29 School of Physics and Astronomy, University of Nottingham, University Park, Nottingham NG7 2RD, UK

30 Institute of Astronomy, University of Cambridge, Madingley Road, Cambridge CB3 OHA, UK

31 UK Astronomy Technology Centre, Blackford Hill, Edinburgh EH9 $3 \mathrm{HJ}, \mathrm{UK}$

32 School of Physical Sciences, The Open University, Milton Keynes MK7 6AA, UK

33 Institute of Astronomy, University of Cambridge, Cambridge CB3 OHA, UK

34 Department of Astrophysics/IMAPP, Radboud University, PO Box 9010, 6500 GL Nijmegen, The Netherlands

35 Kapteyn Astronomical Institute, University of Groningen, Postbus 800, 9700 AV Groningen, The Netherlands

36 Leiden Observatory, Leiden University, PO Box 9513, 2300 RA Leiden, The Netherlands

37 Department for Geophysics, Astrophysics and Meteorology, Institute of Physics, NAWI Graz, Universitätsplatz 5, 8010 Graz, Austria

38 Instituto de Astrofísica e Ciências do Espaço, Faculdade de Ciências da Universidade de Lisboa, 1749-016 Lisboa, Portugal

39 Department of Physics, The University of Hong Kong, Hong Kong SAR, PR China

40 The Laboratory for Space Research, The University of Hong Kong, Hong Kong SAR, PR China

41 Institute of Astronomy and Department of Physics, National Tsing Hua University, Hsinchu 30013, Taiwan

42 ASTRON, The Netherlands Institute for Radio Astronomy, Postbus 2, 7990 AA Dwingeloo, The Netherlands

43 Department of Physics and McGill Space Institute, McGill University, 3600 University Street, Montreal, QC H3A 2T8, Canada 